\title{
Resource Utilization in Clubfoot Management
}

\author{
Matthew A. Halanski MD, Jen-Chen Huang MBChB, \\ Stewart J. Walsh FRACS, Haemish A. Crawford FRACS
}

Published online: 27 January 2009

(C) The Association of Bone and Joint Surgeons 2009

\begin{abstract}
Both private and socialized healthcare systems require treatments to be not only effective, but also costefficient. Although the Ponseti method of clubfoot treatment is effective, its cost-effectiveness has not been demonstrated. We compared the difference in resource use between two prospective cohorts treated for clubfoot by either the Ponseti method or below-knee casting followed by primary surgical release in the socialized healthcare system of New Zealand. Using these cohorts and US billing data, costs of treating these cohorts in the US healthcare system were also calculated. Treatment of initial deformity, recurrences, and complications in both cohorts were included in the final assessment. Twenty-six patients (40 feet) were enrolled in the Ponseti cohort and 29 (46 feet) in the primary surgical cohort. For most patients, the Ponseti method was more cost-effective than the primary surgical treatment in both healthcare systems. The cost of treating both cohorts was lower in the socialized system than in the US healthcare system.
\end{abstract}

\footnotetext{
Each author certifies that he or she has no commercial associations (eg, consultancies, stock ownership, equity interest, patent/licensing arrangements, etc) that might pose a conflict of interest in connection with the submitted article.

Each author certifies that his or her institution has approved or waived approval for the human protocol for this investigation and that all investigations were conducted in conformity with ethical principles of research
}

M. A. Halanski $(\bowtie)$

Helen DeVos Children's Hospital, 1425 Michigan Street NE,

Suite D, Grand Rapids, MI 49503, USA

e-mail: matthew.halanski@devoschildrens.org

J.-C. Huang, S. J. Walsh, H. A. Crawford

Starship Children's Health, Auckland, New Zealand
Level of Evidence: Level II, prognostic study. See the Guidelines for Authors for a complete description of levels of evidence.

\section{Introduction}

Nonoperative primary management of clubfeet has now become the treatment modality of choice for this deformity [10]. The Ponseti and French methods are two of the most common treatments used today. In the United States, the Ponseti method is the more popular method [11, 19, 20]. Proponents of the technique believe it is easy to learn [21], effective at controlling the deformity, available in an office setting, cost-effective, decreases surgical rates [17], and, in comparison with primary surgery, results in better longterm function [7, 16]. Although many papers report the benefit of the Ponseti technique, few well-controlled studies have been published [13-15].

Despite the lack of strong evidence-based medicine for either approach, the switch from surgical release to nonoperative treatment has become the standard of care in the United States [11]. Because of this, enlisting patients into the surgical arm of a well-controlled study is difficult. However, for many years, the main treatment of clubfeet in New Zealand has been primary surgical release. New Zealand is unique in that the prevalence of clubfoot is higher than that in the United States [1, 18]. This environment provided the senior authors (HAC, SJW) of this study a unique opportunity to construct two equivalent cohorts and monitor the differences between treatment methods. In both socialized and private healthcare systems, effective and efficient treatments are required. Although the clinical effectiveness of the Ponseti method has been 
demonstrated [3, 4], its cost-effectiveness has only been implied.

We hypothesized: (1) the Ponseti method of clubfoot treatment would be more cost-effective than below-knee casting followed by primary surgical release; (2) the cost of treating these patients would be less in the New Zealand socialized healthcare system than in the US healthcare system; and (3) fewer nonmonetary resources (hospital days, anesthetics, operative time, antibiotic use, and pain medication) would be used by the Ponseti group. To more accurately determine costs between cohorts, we assumed bilateral cases to cost more than unilateral cases, and thus compared unilateral and bilateral cases separately between cohorts.

\section{Materials and Methods}

In November 2001, after obtaining medical ethical board approval, until January 2005, all 87 infants referred to Starship Children's Health for clubfoot were offered the options for nonsurgical (Ponseti) or surgical management of clubfoot. Families were given the option of randomization or choice of treatment; only nine of these patients' families agreed to randomization. Selection criteria for this review required at least 2 years of clinical followup from the initial casting to be included. In addition, only patients with idiopathic talipes equinovarus were included. Fiftyfive patients, with an average age of 20 days (range, 1034 days) at presentation, with 86 feet met these inclusion criteria. Twenty-six patients (40 feet) were in the Ponseti group and 29 patients (46 feet) were in the below-knee casting and surgery group.

At the time of referral to Starship Children's Health for clubfoot, patients' families met with a dedicated clubfoot nurse coordinator (JED), and treatment options, including either the Ponseti method or below-knee casting and surgical correction, were discussed. The risks and benefits of each method were explained in detail. The family was given informational handouts describing each clinical pathway and was directed to appropriate web sites. Once a clinical pathway was chosen, the patients choosing the Ponseti method were placed under the care of one senior author (HAC) and those in the surgical arm were placed under the care of the other senior author (SJW).

Demographic comparisons between the two prospective cohorts showed no difference in terms of gender, ethnicity, family history, bilaterality, age at first casting, average initial Pirani score (5.0), or years of followup (Table 1). We prospectively collected clinical data at each clinic visit using a templated data sheet (Appendix 1). At the time of presentation, the clubfoot deformities were graded using the validated 6-point scale of Pirani et al. [9]. This was
Table 1. Demographic comparison between cohorts

\begin{tabular}{|c|c|c|c|}
\hline \multirow[t]{2}{*}{ Variable } & \multicolumn{2}{|l|}{ Cohort } & \multirow[t]{2}{*}{$\mathrm{p}$ value } \\
\hline & Ponseti & Surgical & \\
\hline Patients & 26 & 29 & \\
\hline Clubfeet & 40 & 46 & \\
\hline Bilateral & 14 & 17 & 0.8 \\
\hline Gender & & & 1 \\
\hline Male & 18 & 20 & \\
\hline Female & 8 & 9 & \\
\hline Ethnicity & & & 0.6 \\
\hline Polynesian & 17 & 16 & \\
\hline Other & 9 & 13 & \\
\hline Positive family history & 13 & 13 & 0.8 \\
\hline Average Pirani score & 5.2 & 5.2 & 0.9 \\
\hline Average age at first cast & 21 days & 18 days & 0.1 \\
\hline $\begin{array}{l}\text { Average number } \\
\text { pre-(tenotomy/surgical) casts }\end{array}$ & 5 & 11 & $<0.001 *$ \\
\hline Age at tenotomy or surgery & 2.4 months & 6.7 months & $<0.001^{*}$ \\
\hline Average followup & 3.5 years & 3.8 years & 0.2 \\
\hline
\end{tabular}

* Statistically significant.

typically performed by either one of the senior authors or the clubfoot nurse specialist. Other information routinely obtained included ethnicity and family history. Ethnicity was classified as Polynesian or non-Polynesian.

In the Ponseti group, feet were treated at weekly intervals as described by Ponseti [19, 20]. This involved above-knee casting followed by percutaneous tenotomy of the Achilles tendon when indicated and final casting, which remained in place for 3 weeks. All patients in the Ponseti cohort who were compliant with weekly casting required tenotomy. Indications for tenotomy were an inability to dorsiflex the ankle at least $20^{\circ}$ and any case the senior author believed the tendo-Achilles to be tight. One of the senior authors (HAC) managed this cohort as he had been directly taught by spending 1 year with $\mathrm{Dr}$ Ponseti. At the completion of cast treatment, all babies were placed in a foot abduction brace. The open-toed, high-top shoes (MJ Markell Shoe Co, Yonkers, NY) were fitted by the clubfoot nurse coordinator and attached at shoulder width to the Denis Browne bar. Parents were emphatically instructed to ensure full-time brace wear for 3 months followed by night and naptime wear until the age of 2 years [10]. Any problems with casts, brace wear, and brace compliance were noted. As in our previous report, compliance was defined as full-time brace wear for 3 months followed by at least 9 months of nighttime and naptime use [10].

In the surgical group, feet were treated with belowknee plaster casts applied over Elastoplast tape (BSN 
Medical, Auckland, New Zealand). These casts were applied with upward pressure directed under the cuboid. This was believed to help evert the foot and gradually correct the equinus deformity. These below-knee plasters were changed at weekly and biweekly intervals until approximately 6 months of age. When indicated, surgery was then scheduled. The majority of these patients required a posterior or posterior medial release and subsequent plaster changes. The surgical procedures were carried out through a Cincinnati incision [5]. If the patient had isolated hindfoot equinus at the end of casting, a posterior release involving a Z-lengthening of the tendoAchilles, tibiotalar joint release, and posterior subtalar joint release was performed. Patients with both forefoot and hindfoot involvement underwent a full posterior medial release. The subtalar interosseous ligament and deep deltoid ligament were left intact. Patients typically had one subsequent plaster change under anesthesia for a posterior release and two for a posterior medial release. After final plaster removal, patients were followed clinically but required no further brace wear. However, patients who corrected after casting alone (two of 29 patients) were prescribed open-toed, high-top shoes attached at shoulder width to the Denis Browne bar. Patients were then followed at regular intervals as deemed appropriate by the treating surgeon.

Clinical records of all patients in these groups were reviewed. We noted all surgical interventions and complications associated with these deformities. Initial consultation, clinic visits, and all procedures (primary, revision, complications) until last followup were recorded. The average age at followup in the Ponseti group was 3.5 years (range, 2.16-5.5 years) and in the surgical group 3.8 years (range, 2.2-5.7 years). Nonmonetary measures, including operating room time, days in the hospital, number of anesthetics received, and medication required, were reviewed. Using clinic and hospital billing information, the cost of care per foot was then determined. These costs were determined based on the New Zealand public health hospital system. All dollar amounts were then adjusted to US dollars (using a conversion rate of NZ \$0.75 to US \$1.00). Using this clinical data and corresponding CPT codes, the cost of care for these patients as if they had been treated in the United States was calculated. Variables used in determining clinic costs included consultation, casting, foot abduction braces (Markell shoes and Denis Browne Bar), and in-office procedures. Variables used in determining hospital costs included operative time, surgeon and/or anesthetic fees, perioperative care, and number of days in the hospital. Medications are included in the operative and hospital costs in the New Zealand health system and were not billed separately; therefore, these were not included in the cost of care in the US system. Similarly, cost of time in the postanesthesia care unit is combined with day stay or hospital admission costs and not billed separately in the New Zealand system. Therefore, these charges were not entered in the calculations for care within the US healthcare system. For patients awaiting procedures, estimates of operating room time and days in the hospital were based on other patients within the same treatment group undergoing an identical or similar procedure. To determine the end point of clinic visits in the New Zealand system, we used the discontinuation of abduction orthosis in the Ponseti group and the first postoperative visit in those children requiring surgery. This was believed to indicate the end of initial "active treatment" and remove any subjective differences in followup between cohorts. However, any patient found to have a recurrence or complication in either cohort requiring further treatment had these hospital costs included in the final calculations (See Secondary Surgical Costs in Tables 2 and 3). For determinations involving the US system, one visit outside of the 90-day global period was assumed as the end point for routine care (for those feet without recurrences) in those patients requiring surgical management.

Demographic nominal variables (gender, ethnicity, unilateral versus bilateral, and family history) were compared in the two groups by a two-tailed Fisher's exact test, continuous values (age at first casting, initial Pirani score, and age at final followup) by the Student's t test. We determined differences in nonmonetary resources (number of clinic visits, hospital days, anesthetics, operative time, antibiotic doses, and pain medication doses) between the Ponseti and surgical cohorts using the Student's t test. To more accurately assess monetary differences between cohorts, patients within each cohort were further subdivided into unilateral and bilateral cases as well as those with and without recurrences. We then determined monetary differences between each subgroup treated with either the Ponseti or surgical method using the Student's t test. Separate analyses were performed for care given in the New Zealand socialized healthcare system and for the estimated cost of care in the US healthcare system. Finally, a paired Student's t test was used to compare differences in total cost to manage these feet by the Ponseti method or surgery in each of the healthcare systems.

\section{Results}

In the socialized New Zealand healthcare system, we found no difference in clinical costs between cohorts (Table 2). As expected, the surgical group had a higher primary surgical cost than the Ponseti group. We found no difference 


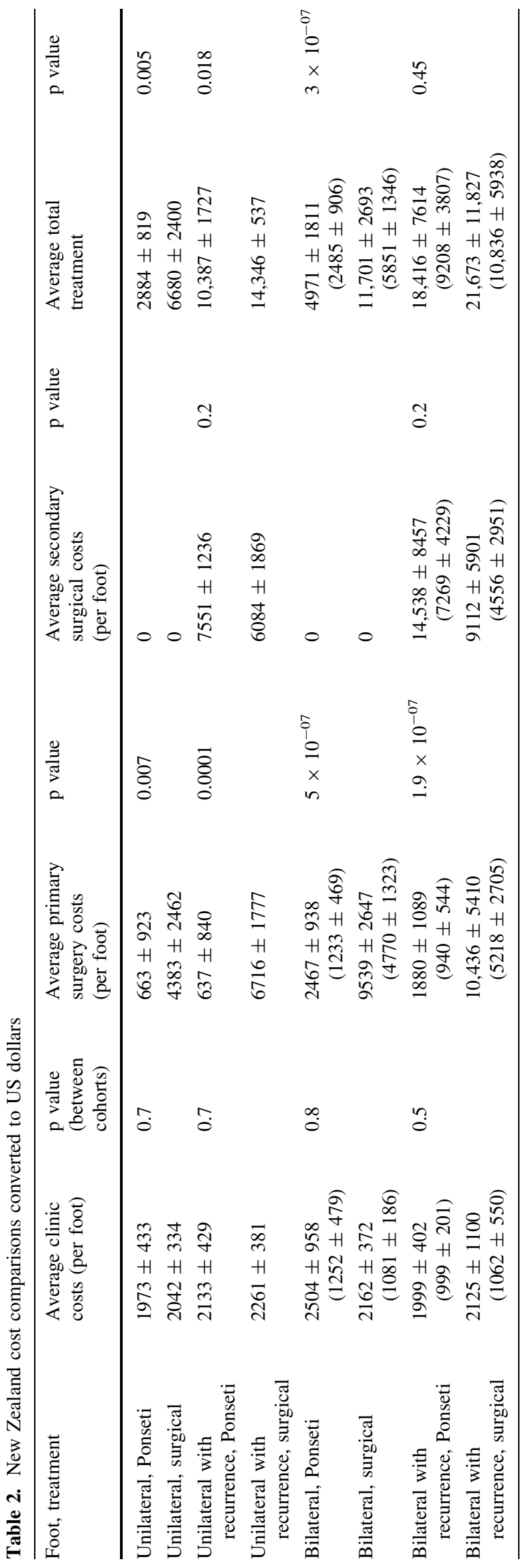

in cost of treating recurrences in either cohort. The total cost of treatment was less for the patients in the Ponseti group with unilateral and bilateral clubfeet and for unilateral clubfeet with recurrences. We observed no difference in total cost for patients with bilateral clubfeet with recurrences (Table 2 ).

Clinical costs in the US healthcare system were similar for unilateral clubfeet for both cohorts. However, there were higher clinic costs for the surgically treated bilateral clubfeet (Table 3). Like in the socialized setting, the surgical group had a higher primary surgical cost than the Ponseti group. The cohorts had similar costs to manage subsequent recurrence and complications. The total cost of treatment was higher in the surgical group than the Ponseti group. Patients with bilateral clubfoot with recurrences had similar costs in the two cohorts (Table 3).

The care for both cohorts was less in the New Zealand socialized healthcare system than in the US healthcare system. All patients except those with unilateral clubfeet and recurrences had a statistically significant lower cost of care in the socialized system (Table 4).

Just as the surgical group was more expensive to treat than the Ponseti group, this cohort used more nonmonetary resources. The surgical cohort required a greater average number of days in the hospital and average pain medication doses than the Ponseti group (Table 5).

\section{Discussion}

The Ponseti method of clubfoot treatment has become popular over the last decade. Others have shown this method to be effective [2, 3, 8, 12] and dramatically decrease the rates of surgical correction [17]. Although presumed, no studies have critically evaluated the costeffectiveness of this method. In this study, we show the cost-effectiveness of the Ponseti method when compared with surgical release in two very different healthcare settings. Importantly, we included the cost to treat recurrences in each group with an average of roughly 3.5 years followup. This is important to consider because up to $40 \%$ of patients treated with the Ponseti method require some further operative treatment to correct a recurrence $[10,16]$. Often, these procedures are ignored when discussing either method's cost-effectiveness. Thus, the purpose of this study was to compare the resource use of the Ponseti method and primary surgical release. We hypothesized the Ponseti method would be more cost-effective than the surgical method. We presumed that as a result of billing differences, treatment in New Zealand would be less expensive than treatment in the United States. Finally, we believed the Ponseti method would use less nonmonetary resources than the surgical treatment. 


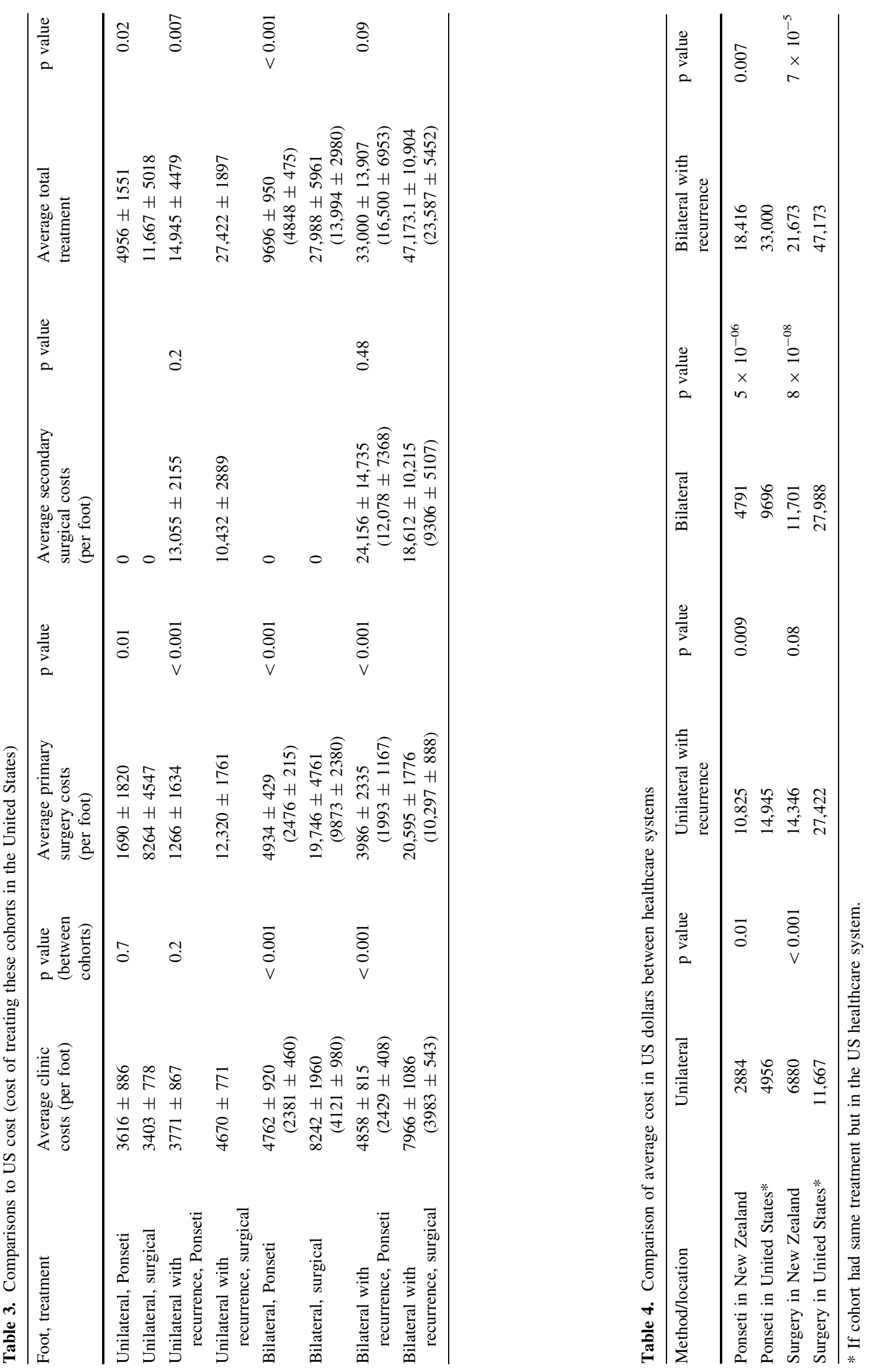


Table 5. Comparison of nonmonetary resource utilization*

\begin{tabular}{|c|c|c|c|c|c|}
\hline \multirow[t]{2}{*}{ Variable } & \multicolumn{2}{|l|}{ Ponseti } & \multicolumn{2}{|l|}{ Surgical } & \multirow[t]{2}{*}{$\mathrm{p}$ value } \\
\hline & Total & Per patient & Total & Per patient & \\
\hline Clinic visits & 297 & 11.0 & 419 & 14.0 & 0.41 \\
\hline Days in hospital & 32 & 1.0 & 76 & 3.0 & 0.04 \\
\hline Anesthetics/trips to operating room & 53 & 2.0 & 80 & 3.0 & 0.47 \\
\hline Operating room time & 54 hours & 124 minutes & 91.9 hours & 190 minutes & 0.054 \\
\hline Antibiotic doses & 91 & 4.0 & 98 & 3.0 & 1 \\
\hline Pain medication doses & 32 & 1.0 & 169 & 6.0 & 0.001 \\
\hline
\end{tabular}

* Values rounded to whole number.

Although these cohorts were prospectively assessed and assembled, treated at the same institution, over the same period of time, this study does have limitations. The short followup time is perhaps the major limitation in this study. With longer followup, more recurrences may occur in both groups, requiring further surgical intervention. However, the majority of recurrences occur while the foot is rapidly growing and recurrences are less common after 3 years of age [6]. Despite this cohort being a "worst case scenario," having less than $50 \%$ brace compliance and $70 \%$ operative tenotomies, the Ponseti method was more cost-effective than the primary surgical method. Despite following these cohorts prospectively, data were collected from clinical charts, electronic records, and operative records and reviewed retrospectively. Because the majority of these patients were citizens of New Zealand and health care is free of charge, costs to nonresidents were based on care given. This is a somewhat artificial cost, but the authors believe this is the most accurate assessment that could be performed with the data available. Similarly, costs of treatment in New Zealand are more often "lumped" rather than itemized as they are in the United States (ie, medications required throughout the hospitalization are included in the hospital stay charge and not billed separately). This likely represented an underestimation of cost in the US healthcare system. Despite this, the authors believe the overall differences and the trends would remain even if dollar amounts were slightly different. Likewise small differences in billings between healthcare systems may have altered final dollar amounts calculated in this study. Three patients had undergone recasting in the Ponseti group and these were billed as clinic visits as is the norm in New Zealand and not as cast applications as they would have been in the US system. Also the one patient in the Ponseti group requiring revision surgery after a post operative infection was not billed for a preoperative visit for her revision tendon transfer in the New Zealand system as this visit was 91 days after her initial tendon transfer and 15 days after her wound debridement and therefore would likely be within the postoperative global period in the US healthcare system. However after taking into account such variations, recalculating billing totals lead to small differences in final dollar amounts but all significant trends remain statistically significant. Finally, as financial markets fluctuate, converting and comparing the cost of care between two systems becomes very difficult. We chose in this study to convert all New Zealand dollars to US dollars using a rounded conversion rate applicable when these data were reviewed. As times change, the values we have calculated may also change, but as stated before, the differences and trends should remain.

Based on our findings, the Ponseti method was more cost-effective in managing the majority of patients in this study. This was true in both the socialized health system of New Zealand as well as in the US healthcare system. The cost of Ponseti treatment is higher than one might expect. Often the technique is considered "low" cost, but in this study, we found the average cost of managing a foot without a recurrence was NZ \$3000 in New Zealand and US $\$ 5000$ in the United States. Some of this cost comes from the fact that over $70 \%$ of the tenotomies were performed in the operating room. This included all bilateral tenotomies and many unilateral tenotomies. Hospital costs could have been decreased if all of these tenotomies had been performed in the office. The senior author (HAC) believes performing the bilateral tenotomies under anesthetic in a more controlled setting is less stressful for the patient and the treating physician, especially when learning this technique. Nearly all unilateral tenotomies are now performed in the office. From this study, we also find the cost of treating a recurrence and/or complication is not insignificant. In New Zealand, the average cost to treat a clubfoot increased from NZ $\$ 3000$ without a recurrence to over NZ $\$ 10,000$ for a foot with a recurrence. Similarly, in the United States, the cost increased from US $\$ 5000$ to nearly US $\$ 15,000$. Thus, managing recurrences or complications can triple the cost of treatment. One major risk factor for recurrence in this New Zealand population is brace intolerance [10]. Other investigators have reported brace wear intolerance as a major risk factor for 
Table 6. Differences in US healthcare reimbursement based on medical insurance

\begin{tabular}{llrrrr}
\hline CPT & Description & Charge & Medicaid & Insurance 1 & Insurance 2 \\
\hline 29450 & Application of leg cast & $\$ 317.90$ & $\$ 82.24$ & $\$ 165.33$ & $\$ 140.57$ \\
27605 & Incision of Achilles tendon, local anesthesia & $\$ 1018.60$ & $\$ 120.78$ & $\$ 267.75$ & $\$ 241.84$ \\
27606 & Incision of Achilles tendon, general anesthesia & $\$ 1256.20$ & $\$ 176.33$ & $\$ 391.62$ & $\$ 353.73$ \\
28262 & Revision of foot and ankle, extensive & $\$ 2871.00$ & $\$ 631.69$ & $\$ 1475.78$ & $\$ 1173.74$ \\
\hline
\end{tabular}

recurrences as well [8]. Like in our previous report, brace intolerance was a major issue in the Ponseti treatment group [10] . Less that $50 \%$ of the patients were actually tolerant of brace wear. In our Ponseti cohort, $40 \%$ of the feet required operative interventions other than tenotomy. With the treatment for these feet costing on average three times more than that for a foot without recurrence, brace intolerance and a high recurrence rate may have a large effect on the cost-effectiveness of the Ponseti method. With such low tolerance and a relatively short (3.5-year) followup, the cost-effectiveness of this method may be lost if more recurrences occur over time and require operative intervention.

Our findings substantiate the general belief the cost of care in the US healthcare system is higher than that in other countries. For both treatment groups, higher costs were seen for management of these disorders in the US system versus that in New Zealand. There are difficulties in "rebilling" these treatments that took place in New Zealand using US billing codes. Many services that are billed separately in the US healthcare system are "lumped" into a global cost in the New Zealand system; thus, determining the exact cost of care of these patients in the US healthcare system is difficult. Examples of these include medications and postanesthesia care unit charges; thus, we did not attempt to include these in our calculations. It is important to note the cost of care in the US health system was based on "billed" costs, not actual money collected for the procedures. Although the "billed" cost of caring for these children was expected to be and was more expensive in the US healthcare system than in New Zealand, the actual money paid out for these procedures would have likely been substantially less than reported in this article (Table 6). Even the highest paying insurance reimbursed less than half the billed cost for each of the procedures. This would have made the actual cost of care in the United States similar to the cost of care in New Zealand. Looking at the reimbursement of the US Medicaid system, the true cost of care for these children in the United States would have likely been considerably less than those in the New Zealand system. Despite the discrepancy between billed and collected monies in the US healthcare system, the New Zealand costs were also determined by billing costs given to patients not covered under the government's socialized system and not actual monies collected; billing costs in both systems were used in this study.

Not only did the Ponseti method save money for the healthcare systems, but resource use was diminished. This was demonstrated by the fewer days spent in a hospital bed in the Ponseti group when compared with the surgical group. Furthermore, patient and family morbidity was less in the Ponseti group as noted by the lower number of anesthetics, days spent in the hospital, and narcotic pain medication required during treatment (Table 5).

Finally, monetary cost is only one aspect of the costeffectiveness of a treatment. The second and more important aspect is the clinical effectiveness of the treatment. The literature documents the effectiveness of the Ponseti method $[3,4,14,16]$. Obviously, the functional results of our cohorts over time will become more important in answering the question, "What is the best way to initially manage idiopathic talipes equinovarus?" From a clinical and cost-effectiveness point of view, the Ponseti method appears be the early answer.

Acknowledgments We thank Jan E. Davison, the clubfoot nurse specialist at Starship Children's Health Auckland, New Zealand, for her time and effort in caring for these children. In addition, we thank Jan for her help in preparing the manuscript. 
Appendix 1

\section{INITIAL CONSULTATION CARD:}

\section{PATUENT + REPRESENTATIVE DETAILS:}

\begin{tabular}{|l|}
\hline PATIENTS FISRT NAME: \\
\hline PATIENTS MMDLE NAME: \\
\hline \\
\hline PATIENTS FAMMY NAME: \\
\hline
\end{tabular}

MAUING ADDRESS:

CONTACT TEL PPHONE:

\section{DAYTIME:}

HOMT:

MOBLE:

CONSENT STATUS:

YES/NO

FAMOLY HISTORY:

\section{ETHNICITY:}

\section{CLINICAL ASSESSMENT:}

- UNI/BI-LATERAL

\section{- PIRANI GRADE}

\begin{tabular}{|l|l|l|}
\hline- lateral surface & - & - \\
\hline$-\quad$ medial crease & - & - \\
\hline$-\quad$ talar head & - & - \\
\hline MFC score: & - & - \\
\hline$-\quad$ posterior heel crease & - & - \\
\hline$-\quad$ empty heel sign & - & - \\
\hline$-\quad$ rigid equinus. & - & - \\
\hline HFC score: & - & - \\
\hline Total score: & - & - \\
\hline
\end{tabular}

RADIOLOGY ASSESSMENT:

\begin{tabular}{|l|l|}
\hline Lateral talocalcaneal angle & - \\
\hline Anteroposterior talocalcaneal angle & - \\
\hline Talocalcaneal index & - \\
\hline $\begin{array}{l}\text { Angle between the longitudinal axis of } \\
\text { calcaneus and the fifth metatarsal }\end{array}$ & - \\
\hline
\end{tabular}

TREATMENT REGEIME:

SURGEON:

TECHNIQUE:

COMMENTS (to include calf circumference, foot length and circumference, skin fold thickness, and range of motion details): 


\section{References}

1. Beals RK. Club foot in the Maori: a genetic study of 50 kindreds. N Z Med J. 1978;88:144-146.

2. Colburn M, Williams M. Evaluation of the treatment of idiopathic clubfoot by using the Ponseti method. J Foot Ankle Surg. 2003;42:259-267.

3. Cooper DM, Dietz FR. Treatment of idiopathic clubfoot. A thirtyyear follow-up note. J Bone Joint Surg Am. 1995;77:1477-1489.

4. Cosma D, Vasilescu D, Vasilescu D, Valeanu M. Comparative results of the conservative treatment in clubfoot by two different protocols. J Pediatr Orthop B. 2007;16:317-321.

5. Crawford AH, Marxen JL, Osterfeld DL. The Cincinnati incision: a comprehensive approach for surgical procedures of the foot and ankle in childhood. J Bone Joint Surg Am. 1982;64:1355-1358.

6. Dietz FR. Treatment of a recurrent clubfoot deformity after initial correction with the Ponseti technique. Instr Course Lect. 2006;55: 625-629.

7. Dobbs MB, Nunley R, Schoenecker PL. Long-term follow-up of patients with clubfeet treated with extensive soft-tissue release. J Bone Joint Surg Am. 2006;88:986-996.

8. Dobbs MB, Rudzki JR, Purcell DB, Walton T, Porter KR, Gurnett CA. Factors predictive of outcome after use of the Ponseti method for the treatment of idiopathic clubfeet. J Bone Joint Surg Am. 2004;86:22-27.

9. Flynn JM, Donohoe M, Mackenzie WG. An independent assessment of two clubfoot-classification systems. J Pediatr Orthop. 1998;18:323-327.

10. Haft GF, Walker CG, Crawford HA. Early clubfoot recurrence after use of the Ponseti method in a New Zealand population. J Bone Joint Surg Am. 2007;89:487-493.

11. Heilig MR, Matern RV, Rosenzweig SD, Bennett JT. Current management of idiopathic clubfoot questionnaire: a multicentric study. J Pediatr Orthop. 2003;23:780-787.
12. Herzenberg JE, Radler C, Bor N. Ponseti versus traditional methods of casting for idiopathic clubfoot. J Pediatr Orthop. 2002;22:517-521.

13. Ippolito E, Farsetti P, Caterini R, Tudisco C. Long-term comparative results in patients with congenital clubfoot treated with two different protocols. J Bone Joint Surg Am. 2003;85:12861294.

14. Ippolito E, Fraracci L, Caterini R, Di Mario M, Farsetti P. A radiographic comparative study of two series of skeletally mature clubfeet treated by two different protocols. Skeletal Radiol. 2003;32:446-453.

15. Ippolito E, Mancini F, Di Mario M, Farsetti P. A comparison of resultant subtalar joint pathology with functional results in two groups of clubfoot patients treated with two different protocols. J Pediatr Orthop B. 2005;14:358-361.

16. Laaveg SJ, Ponseti IV. Long-term results of treatment of congenital club foot. J Bone Joint Surg Am. 1980;62:23-31.

17. Morcuende JA, Dolan LA, Dietz FR, Ponseti IV. Radical reduction in the rate of extensive corrective surgery for clubfoot using the Ponseti method. Pediatrics. 2004;113: $376-380$

18. New Zealand Government. Statistics in New Zealand. Ethnic group for the census usually resident population count 2001. Available at: www.stats.govt.nz. Accessed November 3, 2006.

19. Ponseti IV. Treatment of congenital club foot. J Bone Joint Surg Am. 1992;74:448-454.

20. Ponseti IV. The ponseti technique for correction of congenital clubfoot. J Bone Joint Surg Am. 2002;84:1889-1890.

21. Tindall AJ, Steinlechner CW, Lavy CB, Mannion S, Mkandawire $\mathrm{N}$. Results of manipulation of idiopathic clubfoot deformity in Malawi by orthopaedic clinical officers using the Ponseti method: a realistic alternative for the developing world? J Pediatr Orthop. 2005;25:627-629. 